\title{
S-1-containing chemotherapy for patients with non-small-cell lung cancer: A population-based observational study by the Ibaraki thoracic integrative (POSITIVE) research group
}

\author{
MASAHARU INAGAKI ${ }^{1}$, YOKO SHINOHARA ${ }^{1}$, TAKAYUKI KABURAGI ${ }^{2}$, TAKEO ENDO $^{3}$, SHINSUKE HOMMA $^{4}$, \\ NOBUYUKI HIZAWA $^{4}$, KOJI KISHI ${ }^{5}$, HIROYUKI NAKAMURA ${ }^{5}$, KENJI HAYASHIHARA ${ }^{6}$, TAKEFUMI SAITO ${ }^{6}$, \\ KOICHI KURISHIMA ${ }^{7}$, HIROICHI ISHIKAWA ${ }^{7}$, HIDEO ICHIMURA $^{8}$, TAKESHI NAWA $^{8}$, NORIHIRO KIKUCHI ${ }^{9}$, \\ KUNIHIKO MIYAZAKI $^{10}$, TAKAHIDE KODAMA ${ }^{10}$, HIROAKI SATOH ${ }^{11}$ and KINYA FURUKAWA ${ }^{5}$ \\ ${ }^{1}$ Tsuchiura Kyodo General Hospital and Regional Cancer Center, Tsuchiura, Ibaraki 300-0053; \\ ${ }^{2}$ Ibaraki Prefectural Central Hospital and Regional Cancer Center, Kasama, Ibaraki 309-1793; \\ ${ }^{3}$ Mito Medical Center Hospital, Mito, Ibaraki 311-3193; ${ }^{4}$ Faculty of Clinical Medicine, University of Tsukuba, \\ Tsukuba, Ibaraki 305-8576; ${ }^{5}$ Tokyo Medical University, Ibaraki Medical Center Hospital, Ami, Ibaraki 300-0395; \\ ${ }^{6}$ Ibaraki Higashi National Hospital, Tokai, Ibaraki 319-1113; ${ }^{7}$ Tsukuba Medical Center Hospital and Regional Cancer Center, \\ Tsukuba, Ibaraki 305-8558; ${ }^{8}$ Hitachi General Hospital, Hitachi, Ibaraki 317-0077; ${ }^{9}$ Kasumigaura Medical Center, Tsuchiura, \\ Ibaraki 300-0812; ${ }^{10}$ Ryugasaki Saiseikai General Hospital, Ryugasaki, Ibaraki 301-0854; ${ }^{11}$ Division of Respiratory \\ Medicine, Mito Medical Center, Institute of Clinical Medicine, University of Tsukuba, Mito, Ibaraki 310-0015, Japan
}

Received August 27, 2015; Accepted March 2, 2016

DOI: $10.3892 / \mathrm{mco} .2016 .826$

\begin{abstract}
To evaluate the efficacy and safety of S-1 monotherapy, S-1-containing combined chemotherapy and S-1 containing chemoradiotherapy for non-small cell lung cancer (NSCLC), a population-based observational study was performed. The efficacy and safety of the chemotherapies were evaluated at 13 institutes in a prefecture of Japan between April 2011 and March 2015. Datasets were obtained from 282 patients with NSCLC. For either wild-type or mutated epidermal growth factor receptor (EGFR), these three therapy groups generated almost identical response results and toxicity profiles as those in previously reported clinical trials, although the present study appeared to have slightly lower survival rates compared with those in the previous clinical trials. This may be due to the inclusion of patients in poor condition, and S-1 therapy being administered in the second, or later, line of therapy. In conclusion, the present study has confirmed that S-1-containing chemotherapy is effective against wild- and mutated-type EGFR NSCLC, and it is also tolerable in clinical practice.
\end{abstract}

Correspondence to: Professor Hiroaki Satoh, Division of Respiratory Medicine, Mito Medical Center, Institute of Clinical Medicine, University of Tsukuba, Miya-machi 3-2-7, Mito, Ibaraki 310-0015, Japan

E-mail: hirosato@md.tsukuba.ac.jp

Key words: S-1, non-small cell lung cancer, observational study, population-based

\section{Introduction}

In current clinical practice, epidermal growth factor receptor-tyrosine kinase inhibitor (EGFR-TKI) is the treatment of choice for patients with advanced non-small cell lung cancer (NSCLC) who possess the EGFR mutation. On the other hand, platinum-based doublet regimens with third-generation agents continues to be the standard of care for those with the EGFR wild-type tumor, or who are of unknown EGFR status. S-1, one of the third-generation agents, is an oral fluoropyrimidine consisting of tegafur (a prodrug of fluorouracil), 5-chloro-2,4-dihydropyrimidine (CDHP) and potassium oxonate. CDHP is an inhibitor of dihydropyrimidine dehydrogenase, which is the rate-limiting enzyme for the degradation of fluorouracil (1). S-1 was shown to produce an active response as a single agent for metastatic NSCLC with minimal toxicity $(2,3)$. S-1 has been launched for use as a first-line chemotherapy (4) and a second or third-line chemotherapy (5) in advanced stages of the disease, and chemoradiotherapy for stage III (6).

In spite of these advances in clinical practice, no population-based study on S-1-containing chemotherapy for patients with NSCLC, including elderly patients, has yet been conducted. A major goal of clinical trials is to identify treatment modalities that are able to improve the survival rate and be extrapolated into general clinical practice. Cancer survival statistics are predominantly derived from institutional series or clinical trials, and notably, for such studies, populations of patients are selected. Trials tend to be restrictive with respect to the eligibility criteria, and this, in combination with possible referral and investigator screening biases, results in the inclusion of a more favorable prognostic group 
Table I. Patient characteristics.

\begin{tabular}{|c|c|c|c|c|c|c|}
\hline \multirow[b]{2}{*}{ Parameter } & \multicolumn{2}{|c|}{ S-1-containing monotherapy } & \multicolumn{2}{|c|}{$\begin{array}{c}\text { S-1-containing } \\
\text { combined chemotherapy }\end{array}$} & \multicolumn{2}{|c|}{$\begin{array}{l}\text { S-1-containing } \\
\text { chemoradiotherapy }\end{array}$} \\
\hline & EGFR (-) & EGFR (+) & EGFR (-) & EGFR (+) & EGFR (-) & EGFR (+) \\
\hline No. of patients & 140 & 24 & 73 & 11 & 31 & 3 \\
\hline Age, median (year) & 68 & 67 & 70 & 76 & 68 & 65 \\
\hline Gender (M:F) & $106: 34$ & $12: 12$ & $65: 8$ & $5: 6$ & $26: 5$ & $1: 2$ \\
\hline \multicolumn{7}{|l|}{ Pathology (\%) } \\
\hline $\mathrm{AD}$ & $78(55.7)$ & $21(87.5)$ & $13(17.8)$ & $11(100)$ & $16(51.6)$ & $3(100)$ \\
\hline Others & $62(44.3)$ & $3(12.5)$ & $60(82.2)$ & $0(0)$ & $15(48.8)$ & $0(0)$ \\
\hline BSA, median $\left(m^{2}\right)$ & 1.53 & 1.50 & 1.58 & 1.38 & 1.60 & 1.57 \\
\hline \multicolumn{7}{|l|}{ PS $(\%)$} \\
\hline $0-1$ & $89(63.6)$ & $14(56.3)$ & $49(58.3)$ & $7(63.6)$ & $23(74.2)$ & $3(100)$ \\
\hline $2-3$ & $51(36.4)$ & $10(43.7)$ & $24(41.7)$ & $4(36.4)$ & $8(25.8)$ & $0(0)$ \\
\hline \multicolumn{7}{|c|}{$\begin{array}{l}\text { No. of rounds of } \\
\text { prior chemotherapy (\%) }\end{array}$} \\
\hline $0-1$ & $65(39.6)$ & $3(12.5)$ & $58(79.5)$ & $3(27.3)$ & $28(90.3)$ & $3(100)$ \\
\hline$\geq 2$ & $75(60.4)$ & $21(87.5)$ & $15(20.5)$ & $8(72.7)$ & $3(9.7)$ & $0(0)$ \\
\hline
\end{tabular}

EGFR, epidermal growth factor receptor; M:F, male:female; AD, adenocarcinoma; BSA, body surface area; PS, performance status.

of patients than would be expected in the general population of patients with NSCLC. These selected populations may create a potentially artificial set of treatment standards and outcome expectations. On the other hand, population-based studies exclude selection and referral biases. Several studies have examined this issue in certain cancer types (7-14). In the present study, a population-based observational study was performed to evaluate the efficacy and safety of S-1-containing chemotherapy for patients with NSCLC in a prefecture in Japan.

\section{Patients and methods}

Ibaraki Prefecture in Japan covers an area of 6,095 $\mathrm{km}^{2}$ and has a population of 3 million. This retrospective population-based study included patients with NSCLC who had received S-1 chemotherapy at 16 hospitals in Ibaraki Prefecture between November 2009 and August 2011. All patients were required to have had a pathological or cytological diagnosis of NSCLC. Pathological diagnosis of lung cancer was defined according to the World Health Organization classification. A tumor-lymph node-metastasis (TNM) staging procedure, using head computed tomography (CT; performed on an Aquillion 64 CT scanner; Toshiba, Tokyo, Japan) or magnetic resonance imaging (performed using a MAGNETOM Skyra 3T; Siemens Healthcare Japan, Tokyo, Japan), bone scans, and ultrasonography (performed using the ARIETTA 70 system; Hitachi-Aloka Medical, Tokyo, Japan) and/or CT of the abdomen, was performed for all patients prior to starting the S-1 treatment. Eligible patients were identified in the clinical database of each hospital, and the following information was extracted from their data: Patient demographics at the time of S-1 therapy (age, gender, smoking history, histology and stage) and objective tumor response. The tumor response was evaluated according to the Response Evaluation Criteria in Solid Tumors (RECIST). The performance status (PS) was assigned according to the Eastern Cooperative Oncology Group scale (Common Toxicity Criteria, Version 2.0; published on April 30, 1999). Toxicity was graded according to the National Cancer Institute Common Toxicity Criteria, version 3.0 (15).

According to the three treatment methods (S-1 monotherapy, S-1-containing combined chemotherapy and S-1-containing chemoradiotherapy) and the two EGFR mutation statuses (wild-type and unknown EGFR, and mutated-type EGFR), the patients were divided into six groups: Monotherapy-EGFR (-), monotherapy-EGFR (+), combined chemo-EGFR (-), combined chemo-EGFR (+), chemoradiotherapy-EGFR (-) and chemoradiotherapy-EGFR (+). Taking the EGFR mutation status into consideration, the response rate, the time to treatment failure (TTF), and overall survival (OS) following the initiation of S-1 therapy in each treatment group with or without the EGFR mutation were evaluated.

The present observational study conformed to the Ethical Guidelines for Clinical Studies issued by the Ministry of Health, Labor and Welfare of Japan. The ethical approval for this study was obtained from IRB in the Mito Medical Center, University of Tsukuba (nos. 13-16).

Differences in proportions between two independent groups were compared using the Chi-squared test. $\mathrm{P}<0.05$ was considered to indicate a statistically significant value. The probability of survival was estimated using the Kaplan-Meier method, and the data were compared using the log-rank test. In multivariate analysis, Cox's proportional model was used. 
Table II. S-1 therapy.

\begin{tabular}{|c|c|c|c|c|c|c|}
\hline \multirow[b]{2}{*}{ Parameter } & \multicolumn{2}{|c|}{ S-1-containing monotherapy } & \multicolumn{2}{|c|}{$\begin{array}{c}\text { S-1-containing } \\
\text { combined chemotherapy }\end{array}$} & \multicolumn{2}{|c|}{$\begin{array}{c}\text { S-1-containing } \\
\text { chemoradiotherapy }\end{array}$} \\
\hline & EGFR (-) & EGFR (+) & EGFR (-) & $\operatorname{EGFR}(+)$ & EGFR (-) & $\operatorname{EGFR}(+)$ \\
\hline \multicolumn{7}{|l|}{ Initial dose of S-1 (\%) } \\
\hline$\leq 100 \mathrm{mg}$ & $81(49.4)$ & $14(58.3)$ & $38(52.1)$ & $9(81.8)$ & $12(38.7)$ & $0(0)$ \\
\hline$>100 \mathrm{mg}$ & $59(50.6)$ & $10(41.7)$ & $35(47.9)$ & $92(18.2)$ & $19(61.3)$ & $0(0)$ \\
\hline $\begin{array}{l}\text { Initial dose } / \mathrm{BSA} \\
\text { median }\left(\mathrm{mg} / \mathrm{m}^{2}\right)\end{array}$ & 68.6 & 71.9 & 70.8 & 72.5 & 71.4 & 77.9 \\
\hline Gender (M:F) & $106: 34$ & $12: 12$ & $65: 8$ & $5: 6$ & $26: 5$ & $1: 2$ \\
\hline \multicolumn{7}{|l|}{ Prescription (\%) } \\
\hline $2 \mathrm{Wks}$ taking, $2 \mathrm{Wks}$ rest & $56(40)$ & $6(25)$ & $36(49.3)$ & $1(9.1)$ & $23(74.2)$ & $1(33.3)$ \\
\hline Others & $84(60)$ & $18(75)$ & $37(50.7)$ & $10(90.9)$ & $8(25.8)$ & $2(66.7)$ \\
\hline BSA, median $\left(\mathrm{m}^{2}\right)$ & 1.53 & 1.50 & 1.58 & 1.38 & 1.60 & 1.57 \\
\hline \multicolumn{7}{|l|}{ Dose reduction of S-1 (\%) } \\
\hline Present & $7(5)$ & $4(16.7)$ & $9(12.3)$ & $1(9.1)$ & $7(22.6)$ & $0(0)$ \\
\hline Absent & $133(95)$ & $20(83.3)$ & $64(87.7)$ & $10(90.9)$ & $24(77.4)$ & $3(100)$ \\
\hline
\end{tabular}

EGFR, epidermal growth factor receptor; M:F, male:female; BSA, body surface area; Wks, weeks.

Table III. Response and survival rates of S-1 therapy.

\begin{tabular}{|c|c|c|c|c|c|c|}
\hline \multirow[b]{2}{*}{ Parameter } & \multicolumn{2}{|c|}{ S-1-containing monotherapy } & \multicolumn{2}{|c|}{$\begin{array}{l}\text { S-1-containing } \\
\text { combined chemotherapy }\end{array}$} & \multicolumn{2}{|c|}{$\begin{array}{l}\text { S-1-containing } \\
\text { chemoradiotherapy }\end{array}$} \\
\hline & EGFR (-) & $\operatorname{EGFR}(+)$ & EGFR (-) & $\operatorname{EGFR}(+)$ & EGFR (-) & $\operatorname{EGFR}(+)$ \\
\hline Response rate (\%) & 6.7 & 4.2 & 24.7 & 0 & 58.1 & 0 \\
\hline Disease control rate $(\%)$ & 50.7 & 45.8 & 69.9 & 54.5 & 83.9 & 66.7 \\
\hline Time to failure, months & 2 & 2 & 3 & 3 & 8 & 8 \\
\hline BSA, median $\left(\mathrm{m}^{2}\right)$, months & 10 & 8 & 13 & 28 & 28 & 13 \\
\hline
\end{tabular}

EGFR, epidermal growth factor receptor; BSA, body surface area.

\section{Results}

Patient characteristics. Data sets were obtained from 282 patients with NSCLC (Table I). These patients were divided into $164(58.2 \%)$ in the monotherapy group [140 EGFR (-) and 24 EGFR (+)], 84 (29.8\%) in the S-1-containing combined chemotherapy group [73 EGFR (-) and 11 EGFR (+)], and $34(12.1 \%)$ in the S-1-containing chemoradiotherapy group [31 EGFR (-) and 3 EGFR (+)]. The median age of each treatment group was $\sim 68-70$ years. The $S-1$ monotherapy and S-1-containing chemoradiotherapy groups had a higher proportion of adenocarcinoma compared with that of the S-1-containing combined chemotherapy group, respectively $(\mathrm{P}=0.0001$ and $\mathrm{P}=0.0005)$. For the EGFR (-) patients, the monotherapy group had a higher proportion of patients with prior chemotherapy $(>1)$ than other two therapy groups $(\mathrm{P}=0.0001$ and $\mathrm{P}=0.0001$, compared with the S-1-containing combined chemotherapy and S-1-containing chemoradiotherapy groups, respectively). For the EGFR (+) patients, the S-1 monotherapy group had a higher proportion of patients with prior chemotherapy $(>1)$ compared with that in the $\mathrm{S}-1$-containing chemoradiotherapy group $(\mathrm{P}=0.0064)$. In the S-1 monotherapy and S-1-containing combined chemotherapy groups, patients with the wild-type EGFR had a higher proportion of patients with prior chemotherapy $(>1)$ compared with those in the S-1 monotherapy and S-1-containing combined chemotherapy groups who had received $0-1$ rounds of chemotherapy $(\mathrm{P}=0.0001$ and $\mathrm{P}=0.0003$, respectively).

$S-1$ therapy. Table II shows the initial dose (ID) of S-1 (ID-S-1), the ID-S-1/body surface area (BSA) of the patients, the proportion of patients administered with a drug regimen of 'twice daily for 14 consecutive days followed by a 2-week rest period', and the proportion of patients who experienced dose reduction during the course of the clinical treatment. For the EGFR (-) patients, the S-1-containing combined chemoradiotherapy group had a higher ID/BSA compared with that in the $\mathrm{S}-1$ monotherapy group ( 71.4 vs. $68.6, \mathrm{P}=0.0290)$. In 





the $\mathrm{S}-1$ containing monochemotherapy group, patients with EGFR (+) had a higher ID/BSA compared with those with EGFR (-) treated with S-1-containing S-1 monotherapy (71.9 vs. 68.6; $\mathrm{P}=0.0173$ ). The $\mathrm{S}$-1-containing chemoradiotherapy group had a higher proportion of patients in the twice daily for 14 consecutive days followed by a 2-week rest period' category compared with those in other two therapy groups ( $\mathrm{P}=0.0004$ and $\mathrm{P}=0.0090$, respectively). The $\mathrm{S}-1$-containing chemoradiotherapy group had a higher proportion of patients with dose reduction during the course of the clinical treatment compared with that in the monotherapy group $(\mathrm{P}=0.0104)$.

Response and survival rates in the $S-1$ monotherapy group. For the 140 EGFR (-) patients, the response rate [RR, equal to the complete response $(\mathrm{CR})+$ partial response $(\mathrm{PR})]$, the disease control rate [DCR, equal to the $\mathrm{CR}+\mathrm{PR}+$ stable disease (SD)], the TTF and OS following the initiation of S-1 therapy were $6.7 \%, 50.7 \%, 2$ months and 10 months respectively (Table III). For the 24 EGFR (+) patients, these parameters were $4.2 \%, 45.8 \%, 2$ months and 8 months, respectively (Table III). No statistical differences in these parameters were identified between patients with or without the EGFR mutation status.

Response and survival rates in the $S$-1-containing combined chemotherapy group. For the 73 EGFR (-) patients, the RR, DCR, TTF and OS following the initiation of S-1 therapy were $24.7 \%, 69.9 \%, 3$ months and 13 months, respectively (Table III). For the 11 EGFR (+) patients, the parameters were 0\%,54.5\%, 3 months and 28 months, respectively (Table III). OS following the initiation of the S-1 therapy in patients with EGFR (-) was lower compared with that in patients with EGFR (+) $(\mathrm{P}=0.0041)$.

Response and survival rates in the S-1-containing chemoradiotherapy group. For the 31 EGFR (-) patients, the RR, DCR, TTF and OS following the initiation of S-1 therapy were $58.1 \%, 83.9 \%, 8$ months and 28 months, respectively (Table III). For the 3 EGFR (+) patients, these parameters were $0 \%, 66.7 \%, 8$ months and 13 months, respectively (Table III). No statistical differences in these parameters were identified between patients with or without the EGFR mutation status.

Toxicity in the three therapy groups. Toxicities are shown in Table IV. The most common toxicities were hematological, gastrointestinal and skin toxicities. Esophagitis and pulmonary toxicity were observed in a patient in the S-1-containing chemoradiotherapy group. No treatment-associated mortalities were recorded.

Uni-and multivariate analysis. To identify the prognostic factors, uni- and multivariate analysis were analyzed using the variables below in the three therapy groups: Age $(<70$ or $\geq 70$ years), gender, pathology (adenocarcinoma, others), PS $(0-1, \geq 2)$, the TNM stage (IIIA-B, IV), number of rounds of prior chemotherapy ( $0-1, \geq 2$ ), ID (up to $100 \mathrm{mg}, \geq 100 \mathrm{mg}$ ), ID/BSA (70 mg/m $\mathrm{m}^{2}$ or more, $<70 \mathrm{mg} / \mathrm{m}^{2}$ ) and dose reduction (absent, present). In the univariate analysis, statistical differences were identified for PS $(\mathrm{P}=0.0011)$, prior chemotherapy
$(\mathrm{P}=0.0397)$, ID $(\mathrm{P}=0.0474)$ in the $\mathrm{S}-1$ monotherapy group with EGFR (-), although PS was the only prognostic factor shown to be statistically different in the multivariate analysis $(\mathrm{P}=0.0115)$. In the univariate analysis, statistical differences were only identified for pathology $(\mathrm{P}=0.0055)$ in the $\mathrm{S}-1$ monotherapy-EGFR $(+)$ group, and for PS $(\mathrm{P}=0.0008)$ in the S-1-containing combined chemotherapy EGFR (+) group.

\section{Discussion}

The availability of agents that target the EGFR tyrosine kinase has provided promising clinical benefits in specific subpopulations of NSCLC. At present, first- and second-generation EGFR-TKIs are available for clinical use. Even though a strong consensus exists on the use of EGFR-TKI as the treatment in patients with EGFR-mutated tumors, only $~ 10-30 \%$ of patients with NSCLC have mutated EGFR $(16,17)$. Therefore, platinum-based doublet chemotherapy continues to provide the standard of care for those patients with EGFR wild-type tumors or who have unknown EGFR status, and no clear differences were identified in OS between patients with or without the EGFR mutation status. In the present study, therefore, TTF and OS following S-1 therapy were evaluated, taking the EGFR mutation status into consideration for this purpose.

$\mathrm{S}-1$ was shown to produce an active response as a single agent for metastatic NSCLC with minimal toxicity $(2,3,18)$. Kawahara et al (2) reported that S-1 monotherapy had an RR of $22 \%$, median response duration, 3.4 months, and OS of $22 \%$. As a second-line S-1 monotherapy, the RR, progression-free survival (PFS) and OS in two prospective phase II studies were $12.5 \%, 2.5$ months, 8.2 months and 14\%, and 4.2 months and 16.4 months, respectively $(3,19)$. In addition, $\mathrm{S}-1$ has been evaluated in the combined setting with cisplatin (CDDP) or carboplatin (CBDCA) (19,20). A randomized phase III study in chemotherapy-naïve patients was performed to assess whether treatment with S-1 and CDDP was as effective as CDDP and docetaxel (DTX) (19). S-1 and CDDP in combination were not inferior to CDDP and DTX with respect to OS [mean survival time (MST) 16.1 vs. 17.1 months, respectively] (19). The non-inferiority of S-1/CBDCA compared with CBDCA/paclitaxel was confirmed for OS (MST, 15.2 vs. 13.3 months, respectively) (20). As a chemoradiotherapy treatment, two phase II trials of S-1 and CDDP combined with concurrent thoracic radiotherapy were evaluated in patients with locally advanced NSCLC $(6,21)$. The RR, PFS and OS of the trials were $82 \%, 20$ months, and not reached at a follow-up time, and 88\%, 12 months and 33.1 months, respectively $(6,21)$.

In the monotherapy group in the present study, OS following S-1 therapy was lower compared with those in previous clinical trials $(2,3,18)$. This may be due to the inclusion of patients with second-, or a later, line of S-1 monotherapy in the present study. In the S-1-containing combined chemotherapy group, OS following S-1 therapy in EGFR (-) patients was lower compared with that in patients with EGFR (+). This result apparently was associated with the effect of TKIs administered following S-1 therapy. In the S-1-containing chemoradiotherapy group, OS following S-1 therapy appeared to be lower compared with those in the clinical trials previously reported $(6,20)$. This may be due to the inclusion of patients with stage IV cancer (41.2\%) and those with PS 2-3 (23.5\%) in the present study. 
Population-based studies are useful to evaluate the applicability and impact of clinical trials in clinical practice. A burgeoning body of evidence from a number of population-based studies has demonstrated considerable variability in standard treatment and survival rates for patients with lung cancer (7-14). However, no population-based study has been performed on S-1-containing chemotherapy.

In the present era of evidence-based medicine, therapeutic modalities are evaluated within a paradigm in which clinical trial outcomes are extrapolated from a select group to the population as a whole. Although the logic of this approach may be correct, it is very important to be able to evaluate how applicable the results are to the population as a whole. Clinical trials rarely provide an estimate of the number of patients for whom a given therapy may be appropriate, or the denominator of applicability. Therefore, the efficacy and safety of the therapy must be confirmed in a certain number of unselected patients throughout the population.

The present study had certain limitations. The retrospective design, without a large number of patients, limited the extent to which these results may be applied to the population at large. However, the results obtained in our population-based study were comparable with those reported in previously published clinical trials, particularly with respect to the RR, TTF and OS following the initiation of S-1 therapy, and the adverse effects. It seems reasonable that novel treatment modalities should also be evaluated by population-based studies, since clinical trials tend to include highly selected populations that are not representative of oncology patients in general.

\section{References}

1. Shirasaka T, Shimamoto Y, Ohshimo H, Yamaguchi M, Kato T, Yonekura K and Fukushima M: Development of a novel form of an oral 5-fluorouracil derivative (S-1) directed to the potentiation of the tumor selective cytotoxicity of 5-fluorouracil by two biochemical modulators. Anticancer Drugs 7: 548-557, 1996.

2. Kawahara M, Furuse K, Segawa Y, Yoshimori K, Matsui K, Kudoh S, Hasegawa K and Niitani H; S-1 Cooperative Study Group (Lung Cancer Working Group): Phase II study of S-1, a novel oral fluorouracil, in advanced non-small-cell lung cancer. Br J Cancer 85: 939-943, 2001.

3. Shiroyama T, Kijima T, Komuta K, Yamamoto S, Minami S, Ogata Y, Okafuji K, Imamura F, Hirashima T, Tachibana I, et al: Phase II tailored S-1 regimen study of first-line chemotherapy in elderly patients with advanced and recurrent non-small cell lung cancer. Cancer Chemother Pharmacol 70: 783-789, 2012.

4. Okamoto I, Yoshioka H, Morita S, Ando M, Takeda K, Seto T, Yamamoto N, Saka H, Asami K, Hirashima T, et al: Phase III trial comparing oral S-1 plus carboplatin with paclitaxel plus carboplatin in chemotherapy-naive patients with advanced non-small-cell lung cancer: Results of a west Japan oncology group study. J Clin Oncol 28: 5240-5246, 2010.

5. Ono A, Naito T, Murakami H, Takahashi T, Nakamura Y, Tsuya A, Kaira K, Igawa S, Shukuya T, Tamiya A, et al: Evaluation of S-1 as third or further-line chemotherapy in advanced non-small-cell lung cancer. Int J Clin Oncol 15: 161-165, 2010.

6. Ohyanagi F, Yamamoto N, Horiike A, Harada H, Kozuka T, Murakami H, Gomi K, Takahashi T, Morota M, Nishimura T, et al: Phase II trial of S-1 and cisplatin with concurrent radiotherapy for locally advanced non-small-cell lung cancer. Br J Cancer 101: 225-231, 2009.
7. Richardson GE, Thursfield VJ and Giles GG: Reported management of lung cancer in Victoria in 1993: Comparison with best practice. Anti-cancer council of victoria lung cancer study group. Med J Aust 172: 321-324, 2000.

8. Sambrook RJ and Girling DJ: A national survey of the chemotherapy regimens used to treat small cell lung cancer (SCLC) in the United Kingdom. Br J Cancer 84: 1447-1452, 2001.

9. Gregor A, Thomson CS, Brewster DH, Stroner PL, Davidson J, Fergusson RJ and Milroy R; Scottish Cancer Trials Lung Group; Scottish Cancer Therapy Network: Management and survival of patients with lung cancer in Scotland diagnosed in 1995: Results of a national population based study. Thorax 56: 212-217, 2001

10. Lebitasy MP, Hédelin G, Purohit A, Moreau L, Klinzig F and Quoix E: Progress in the management and outcome of small-cell lung cancer in a French region from 1981 to 1994. Br J Cancer 85: 808-815, 2001.

11. Chang GC, Chen KC, Yang TY, Yin MC, Lin CP, Kuo BI and Hsu JY: Activity of gefitinib in advanced non-small-cell lung cancer with very poor performance status. Invest New Drugs 23: 73-77, 2005.

12. Hayashibara K, Satoh H, Shinohara Y, Inagaki M, Kaburagi T, Hashimoto T, Kurishima K, Ishikawa $\mathrm{H}$, Ichimura $\mathrm{H}$, Nawa T, et al: A population-based study of gefitinib in patients with non-small cell lung cancer. Med Oncol 26: 222-227, 2009.

13. Nakamura H, Satoh H, Kaburagi T, Nishimura Y, Shinohara Y, Inagaki M, Endo T, Saito T, Hayashihara K, Hizawa N, et al: Bevacizumab-containing chemotherapy for non-small cell lung cancer patients: A population-based observational study by the Ibaraki thoracic integrative (POSITIVE) research group. Med Oncol 29: 3202-3206, 2012.

14. Kaburagi T, Satoh H, Hayashihara K, Endo T, Hizawa N, Kurishima K, Nishimura Y, Hashimoto T, Nakamura H, Kishi $\mathrm{K}$, et al: Observational study on the efficacy and safety of erlotinib in patients with non-small cell lung cancer. Oncol Lett 5:435-439, 2013.

15. Cancer Therapy Evaluation Program, Common Toxicity Criteria for Adverse Events, Version 3.0, CTCAE Version 3.0, June 10, 2003, http://ctep.cancer.gov, Accessed May 15, 2014.

16. Reck M, Heigener DF, Mok T, Soria JC and Rabe KF: Management of non-small-cell lung cancer: Recent developments. Lancet 382: 709-719, 2013.

17. Chen YM: Update of epidermal growth factor receptor-tyrosine kinase inhibitors in non-small-cell lung cancer. J Chin Med Assoc 76: 249-257, 2013.

18. Totani Y, Saito Y, Hayashi M, Tada T, Kohashi Y, Mieno Y, Kato A, Imizu H, Yoneda Y, Hoshino T, et al: A phase II study of S-1 monotherapy as second-line treatment for advanced non-small cell lung cancer. Cancer Chemother Pharmacol 64: $1181-1185,2009$

19. Katakami N, Gemma A, Sakai H, Kubota K, Nishio M, Inoue A, Okamoto $\mathrm{H}$, Isobe $\mathrm{H}$, Kunitoh $\mathrm{H}$, Takiguchi $\mathrm{Y}$, et al: Randomized phase III trial of S-1 plus cisplatin versus docetaxel plus cisplatin for advanced non-small-cell lung cancer (TCOG0701). J Clin Oncol 30 (Suppl): abstract 7515, 2012.

20. Yoshioka H, Okamoto I, Morita S, Ando M, Takeda K, Seto T, Yamamoto N, Saka H, Atagi S, Hirashima T, et al: Efficacy and safety analysis according to histology for S-1 in combination with carboplatin as first-line chemotherapy in patients with advanced non-small-cell lung cancer: Updated results of the West Japan Oncology Group LETS study. Ann Oncol 24: 1326-1331, 2013

21. Ichinose Y, Seto T, Sasaki T, Yamanaka T, Okamoto I, Takeda K, Tanaka M, Katakami N, Sawa T, Kudoh S, et al: S-1 plus cisplatin with concurrent radiotherapy for locally advanced non-small cell lung cancer: A multi-institutional phase II trial (West Japan Thoracic Oncology Group 3706). J Thoracic Oncol 6: 2069-2075, 2011. 